\title{
Avaliação da disciplina de telenfermagem: uma estratégia de ensino a distância
}

\author{
Solange Cervinho Bicalho Godoy, D.Sc.*, Eliane Marina Palhares Guimarães, D.Sc.**, \\ Lenice de Castro Mendes Villela, D.Sc. ${ }^{* * *}$, Driely Suzy Soares Assis ${ }^{* * * *}$
}

\begin{abstract}
*Sub-coordenadora do projeto Telenfermagem/ EEUFMG/Programa Nacional de Telessaúde do Ministério da Saúde, Subcoordenadora do Projeto de Telenfermagem/PBH-HC-EEUFMG, **Vice-Diretora da Escola de Enfermagem da UFMG Gestão 2010/2014, Coordenadora do Projeto de Telenfermagem/EEUFMG/Programa Nacional de Telessaúde do Ministério da Saúde, Coordenadora do Projeto de Telenfermagem/PBH-HC-EEUFMG, ${ }^{* *}$ Coordenadora da disciplina Estágio Curricular 1 / Internato Rural e Metropolitano da EEUFMG/EEUFMG, ${ }^{* * * *}$ Aluna de graduação do curso de Enfermagem da Escola de Enfermagem da Universidade Federal de Minas Gerais e Bolsista de monitoria do Projeto de Telenfermagem/PROGRAD/ EEUFMG
\end{abstract}

\section{Resumo}

Introdução: No Brasil, a Educação à Distância (EAD) surge recentemente como uma ferramenta estratégica importante, tendo em vista as dificuldades enfrentadas pelos alunos e profissionais, especialmente para prestar assistência à saúde da comunidade urbana e rural. O Projeto de Telenfermagem é parte integrante do Programa Nacional de Telessaúde, promovido pelo Ministério da Saúde com o propósito de apoiar profissionais inseridos na Atençáo Primária à Saúde (APS) que utilizam a ferramenta da EAD. Objetivo: Avaliar a utilização das ferramentas do Projeto de Telenfermagem durante o desenvolvimento da disciplina Estágio Curricular I da Escola de Enfermagem da Universidade Federal de Minas Gerais. Método: Trata-se de um estudo descritivo, transversal, realizado com 23 alunos, matriculados no segundo semestre de 2011 e primeiro semestre de 2012. Para a coleta de dados, utilizou-se formulário semiestruturado e os dados foram analisados por estatística descritiva simples. Resultados: Evidenciaram a importância e o apoio que o projeto oferece ao aluno no processo de aprendizagem nos campos de estágio. Conclusão: A utilização das ferramentas do projeto foi positiva, pois possibilitou uma troca de experiência a partir da discussão teórica e prática entre docentes e discentes da Universidade e os profissionais da APS.

Palavras-chave: educação em saúde, telenfermagem, educação à distância, atenção primária a saúde.

\section{Abstract}

\section{Evaluation of discipline of telenursing: a strategy for distance education}

Introduction: In Brazil, Distance Learning (DL) has recently arisen as important and strategic tool, considering the difficulty faced by students and professionals in offering health assistance to both urban and rural communities. The Telenursing Project is a branch of the National Project of Telehealth, promoted by the Ministry of Health, with the ambition of supporting professionals who are engaged with Primary Health Care (PHC) using the tool EAD. Objective: To evaluate the appliance of instruments provided by the Telenursing Project during the development of the discipline Estágio Curricular I / Internato Rural e Metropolitano of Nursing School of the Federal University of Minas Gerais. 
Method: It is a descriptive transversal study, conducted with 23 students enrolled in the second half of 2011 and first half of 2012. For data collection, we used semi-structured form and data were analyzed by simple descriptive statistics. Results: Highlighted the importance and the support that the project offers to student in the learning process in the field of internship. Conclusion: The tools used in the project were positive; they enabled an exchange of experience from the theoretical and practical discussion among teachers and students of the University and the professional PHC.

Key-words: health education, telenursing, distance learning, primary health care.

\section{Resumen}

\section{Evaluación de la asignatura teleenfermería: una estrategia para la educación a distancia}

Introducción: En Brasil, la Educación a Distancia (EAD) surge recientemente como una herramienta estratégica importante, pensando en las dificultades enfrentadas por alumnos y profesionales, para prestar asistencia de salud de la comunidad urbana y rural. El Proyecto de Teleenfermería, integrante del Programa Nacional de Telesalud, promovido por el Ministerio de la Salud, tiene como propósito apoyar a profesionales de Atención Primaria de la Salud (APS) utilizando la herramienta de EAD. Objetivo: Evaluar la utilización de herramientas de dicho proyecto a lo largo de la asignatura Práctica Curricular I/Internado Rural y Metropolitano de la Escuela de Enfermería de la Universidad Federal de Minas Gerais. Método: Se realizó un estudio descriptivo transversal, realizado con 23 estudiantes matriculados en el segundo semestre de 2011 y primer semestre de 2012. Para la colecta de datos, se utilizó formulario semiestructurado y los datos fueron analizados por estadísticas descriptivas simples. Resultados: Estos resultados ponen de relieve la importancia y el apoyo que el proyecto ofrece al estudiante en el proceso de aprendizaje en el campo de prácticas. Conclusión: El uso de las herramientas del proyecto fue positiva, pues permitió el intercambio de experiencias de la discusión teórica y práctica entre profesores y estudiantes de la Universidad y de la APS profesional.

Palabras-clave: educación en salud, teleenfermería, educación a distancia, atención primaria de salud.

\section{Introdução}

A Educação à Distância (EAD) surge da necessidade da formaçáo e qualificação de um contingente populacional que não tinha acesso e/ou condiçóes de frequentar um estabelecimento de ensino presencial. A sua evolução ocorreu juntamente com as tecnologias desenvolvidas em cada momento histórico, as quais influenciaram não só o ambiente educativo, mas a sociedade como um todo [1].

A evolução da EAD ocorreu inicialmente com a utilizaçáo de materiais impressos, que posteriormente se aliaram ao rádio e a televisão. Com o advento da informática, a EAD também incorporou o computador e, mais recentemente, a tecnologia de multimídias que combina textos, sons, imagens. Essas novas tecnologias de informação e comunicação ampliaram as possibilidades da EAD, tornando-a uma ferramenta de desenvolvimento ao proporcionar a formação de cidadãos, qualificação de pessoal para a demanda do mercado de trabalho, além da possibilidade de utilização de novas tecnologias [1].
A EAD é uma modalidade de educação em que professores e alunos encontram-se em locais diferentes durante o processo de ensino-aprendizagem, sendo entendida como uma estratégia de democratização do acesso à educação [1]. Essa estratégia permite o acesso a um grande número de pessoas a programas educativos, independentemente do tempo e da localização, e é desenvolvida em um ambiente interativo com o apoio de recursos instrucionais. A EAD é influenciada pela compreensão de distância com base na separaçáo espacial (geográfica/ local) entre participantes do processo educacional, sejam estes alunos ou professores [2].

Essa modalidade de aprendizagem pode ser vista como uma forma de superação de lacunas educacionais na qualificação profissional e aperfeiçoamento ou atualização de conhecimentos. Atualmente, vem sendo utilizada como uma modalidade de ensino alternativo, possibilitando que, independentemente da presença física dos participantes no mesmo espaço geográfico, qualquer pessoa adquira o conhecimento sobre o assunto de seu interesse [3]. 
Assim, a interação da educação-tecnologia na modalidade a distância, se justifica para os profissionais da saúde, mediante as dificuldades e até inviabilidade de deslocamentos de seus locais de trabalho, além da possibilidade de poder receber uma capacitação permanente que poderá subsidiar as decisóes e ampliar os conceitos sobre o processo saúde e doença, considerando seus condicionantes e as consequências para as práticas assistenciais, garantindo assim uma maior autonomia e resolutividade na assistência [4].

Como vivemos em um país de dimensóes continentais extensas, a missão de proporcionar uma formação adequada a todos os profissionais do Sistema Único de Saúde (SUS), pelos meios tradicionais, torna-se onerosa e de difícil realização. Dessa forma, a EAD surge como uma forma de transpor esse impasse [3]. Entretanto, não deve ser considerada como substitutiva de outras formas de educação convencional, mas como modalidade educacional alternativa que assegura significativamente o ensino à distância para os profissionais de saúde inseridos no mercado de trabalho [5].

Por ser uma ferramenta de desenvolvimento e de qualificação, essa nova tecnologia de educação foi incorporada ao SUS como modalidade educacional alternativa. Essa tecnologia atua de forma significativa no ensino à distância com o objetivo de refletir e discutir a prática assistencial, capacitando e atualizando os profissionais de saúde de forma permanente para o desenvolvimento das açóes, segundo as necessidades de promoção, prevenção, diagnóstico, tratamento e reabilitação da saúde, fundamentadas nas melhores práticas disponíveis e aplicadas às diversas realidades do Brasil.

A EAD é uma estratégia de ensino-aprendizagem que possibilita o intercâmbio de conhecimentos entre os profissionais e instituiçóes de ensino e pesquisa, além da criação de mecanismos que reduzam as situaçóes de vulnerabilidade e propostas que pressupóem a equidade e a participaçáo e o controle social na gestão das políticas públicas [6].

A Atenção Primária à Saúde (APS) é a política estruturante do SUS, pois ocupa a base da hierarquia dos serviços de saúde. Esta estende-se em todo o território nacional e articula-se aos demais níveis de saúde, organizados regionalmente, de acordo com as complexidades e as necessidades especiais em cada nível de atenção à saúde.

O Programa Nacional de Telessaúde, instituído pelo Ministério da Saúde, responde, parcialmente, a essa proposta de ensino à distância ao disponibilizar uma rede de informação entre os municípios integrantes do programa com os Núcleos de Telessaúde referência, presentes nos centros universitários de cada regiáo dos quatorze estados cadastrados: Amazonas, Ceará, Goiás, Pernambuco, Minas Gerais, Rio de Janeiro, Rio Grande do Sul, Santa Catarina, São Paulo, Tocantins, Mato Grosso do Sul, Espírito Santo, Paraíba e Rio Grande do Norte.

O Projeto Telenfermagem, parte integrante do Programa Nacional de Telessaúde, desenvolvido pela Escola de Enfermagem da Universidade Federal de Minas Gerais (EEUFMG), vem utilizando de forma efetiva a EAD para os profissionais e alunos inseridos na APS. É um projeto de extensão com interface com a pesquisa que tem como prioridade instrumentalizar, de forma ágil e eficiente, os profissionais de saúde, com ênfase na educação permanente, para atenderem as necessidades locais e ou concatenar conhecimentos norteadores de uma prática profissional engajada no mercado de trabalho, especialmente nos municípios do interior de Minas Gerais.

Para a operacionalização do projeto, utilizam-se tecnologias de comunicação e informação de fácil acesso, manuseio e de custo baixo. Os equipamentos de hardware necessários são de tecnologia simples e compreendem um microcomputador com multimídia e webcam.

As vantagens da utilização da ferramenta do Projeto Telenfermagem estáo na facilidade de acesso a protocolos sistematizados, pesquisa colaborativa entre centros de ensino, sessóes de segunda opinião, reduçáo do tempo entre o diagnóstico e tratamento, fortalecendo a eficácia e eficiência do serviço de saúde para minimizar os agravos que acometem à população [7].

Para se alcançar e atender as propostas, o Projeto Telenfermagem utiliza dois recursos tecnológicos: a videoconferência temática e a teleconsultoria. A videoconferência é definida como uma conexão entre pessoas em posiçóes geograficamente afastadas, em tempo real, com a finalidade de comunicaçáo, envolvendo áudio, texto e vídeo [8].

A teleconsultoria é uma ferramenta que integra, de forma individualizada, o aluno e ou profissional de saúde inserido na APS, inclusive os especialistas. Caracteriza-se como uma segunda opiniáo, podendo abordar questóes relativas aos problemas de saúde, ao nível de complexidade científica e técnica da medicina, e também aos pro- 
blemas legais e econômicos associados ao exercício da prática profissional. Porém, é importante salientar que a responsabilidade pela atençáo à saúde e pelo cuidado com o usuário é do profissional que o atende diretamente ou da equipe da Estratégia de Saúde da Família (ESF).

A teleconsultoria possui duas modalidades operacionais: on-line, em que o profissional apresenta um caso clínico em tempo real para o especialista e off-line na qual o profissional envia o resumo do caso e o especialista responde via sistema. Nas duas modalidades, o profissional recebe orientaçóes quanto ao diagnóstico, terapia, laudo, condutas gerais ou propedêuticas $[9,10]$.

Entende-se que a utilização dos recursos tecnológicos disponíveis, videoconferências, teleconsultorias online e off-line e cursos de educação à distância, é uma estratégia que possibilita a aproximação professor e aluno durante as orientaçôes do estágio. Ao mesmo tempo oferece ao profissional da APS a oportunidade de receber uma capacitaçáo e ou solicitar uma segunda opiniáo. Essas atividades consistem em um processo interativo que foca a transmissão de um conhecimento de forma dirigida para a construção do raciocínio com referência no problema encaminhado a um profissional especializado [11].

Com essa disponibilidade tecnológica e, tendo em vista a importância do ensino e educação na formação profissional de enfermeiros, optou-se pela utilizaçáo do recurso tecnológico disponibilizado pelo Projeto Telenfermagem para os alunos da disciplina Estágio Curricular I - Internato Rural e Metropolitano - do Curso de Graduaçáo em Enfermagem da EEUFMG. Essa disciplina, com uma carga horária de 450 horas práticas, tem como propósito inserir o estudante na APS nos municípios do interior e capital do Estado de Minas Gerais, para desenvolver competências nas dimensóes assistenciais, gerenciais, educativas e de investigação em enfermagem.

O uso da tecnologia de comunicação e informação é uma ferramenta alternativa relevante para a capacitaçáo, considerando que os alunos estáo em um momento de construção e aprimoramento de conhecimentos, habilidades e atitudes essenciais ao exercício profissional da enfermagem. Nesse sentido, o aluno tem a oportunidade de vivenciar diferentes realidades com um suporte tecnológico para aperfeiçoamento e desenvolvimento de açóes que respondam às demandas locais de saúde dos municípios onde estáo inseridos. Destaca-se ainda a importância da participação dos profissionais de saúde neste processo de educaçáo permanente o que propicia uma melhor assistência para a comunidade, além da aproximação do docente com o recurso tecnológico no processo de ensino aprendizagem [10].

Com essa iniciativa, a disciplina Estágio Curricular I em parceria com o Projeto de Telenfermagem/EEUFMG busca contribuir para a formação de recursos humanos e o desenvolvimento das habilidades e competências necessárias para que os alunos possam intervir no processo saúde-doença junto às comunidades. Essa estratégia de integraçáo ensino-serviço no contexto da atenção primária contribui para o processo de formaçáo do aluno na área da saúde, em consonância com os pressupostos do SUS.

Diante desse contexto, o presente estudo tem como objetivo avaliar a utilização das ferramentas do Projeto Telenfermagem no processo de capacitaçáo dos alunos da disciplina Estágio Curricular I / EEUFMG, para o desenvolvimento das diversas competências que compóem o perfil do profissional enfermeiro na APS, nos municípios do Estado de Minas Gerais, cadastrados pelo Programa Nacional de Telessaúde.

\section{Material e métodos}

Trata-se de um estudo descritivo, transversal tendo como objetivo avaliar a utilização das ferramentas do Projeto Telenfermagem pelos alunos da disciplina Estágio curricular I da EEUFMG, no segundo semestre de 2011 e primeiro semestre de 2012.

A coleta dos dados ocorreu ao final de cada semestre letivo da disciplina, nos dois semestres letivos, com a aplicação de um questionário semiestruturado que contemplava questóes referentes à identificaçáo do aluno, local do estágio e a sua avaliaçáo relacionada ao emprego das ferramentas do Projeto Telenfermagem.

Para participar do referido projeto, os alunos e docentes receberam um treinamento, no início de cada semestre letivo, quando foi demonstrado o manuseio das ferramentas do Projeto Telenfermagem: videoconferências e teleconsultorias off-line e online. Ressalta-se, neste momento, a responsabilidade e o compromisso do aluno na utilização do sistema em relaçáo à segurança dos dados, ao sigilo das informaçóes e à postura ética. Foram aplicados 
23 questionários, dos quais 15 no segundo semestre de 2011 e 8 no primeiro semestre de 2012.

O questionário foi norteado pelas seguintes perguntas: Você utilizou o recurso da teleconsultoria/videoconferência durante o estágio curricular I?; Na sua opiniáo, a teleconsultoria/videoconferência contribui para a troca de informaçóes entre os alunos, docentes e profissionais?; Como você avalia os recursos da ferramenta de teleconsultoria/ videoconferência?; Você encontrou problemas na utilização das ferramentas?; $\mathrm{O}$ que você sugere para aperfeiçoar a ferramenta?

Para análise dos dados, utilizou-se de uma planilha eletrônica do software Excel. Os dados foram tabulados e apresentados por uma frequência relativa e absoluta, para uma análise estatística descritiva.

Os critérios de inclusão definidos constituíram na participação dos alunos nas nove videoconferências realizadas a cada semestre e/ou a solicitação de teleconsultorias durante cada semestre letivo. Excluíram-se aqueles que náo utilizaram as ferramentas do projeto Telenfermagem.

Após a concordância em participar do estudo, os sujeitos da pesquisa assinaram o Termo de Consentimento Livre e Esclarecido contendo informaçóes referentes ao objetivo, metodologia, análise dos dados e à divulgaçáo dos resultados, de forma a preservar a confidencialidade e privacidade, salvaguardando-se o anonimato dos participantes conforme as normas contidas na Resolução 196/96 do Conselho Nacional de Saúde sob o parecer $n^{\circ}$ ETIC 0514.0.203.000-10 do Comitê de Ética em Pesquisa da UFMG-COEP aprovado em 14 de março de 2011.

\section{Resultados e discussão}

A amostra constou de 23 alunos, que cursaram a disciplina Estágio Curricular I, durante o segundo semestre de 2011 e o primeiro semestre de 2012.

No segundo semestre de 2011, observou-se que as participaçóes nas videoconferências ocorreram em duas ou mais vezes com um percentual de $73,3 \%$. No primeiro semestre de 2012, a frequência de duas ou mais vezes nas atividades de videoconferências ocorreu em 87,5\%. Com esses dados, pode-se inferir que há uma participação mais efetiva com um incremento gradual dos alunos da disciplina do Estágio Curricular I/ EEUFMG no Projeto Telenfermagem. Acredita-se que o processo ensino-aprendizagem se constitui a partir da inserção do aluno no campo de estágio, quando assume efetivamente o processo do cuidado e sente a necessidade de buscar alternativas para sua qualificação na APS.

Considerando que as videoconferências abordam temas que são elencados anteriormente pelos profissionais e alunos inseridos na APS, de acordo com as necessidades cotidianas de seu trabalho, entende-se que as discussóes promovidas a partir das videoconferências possibilitam espaço para a troca de informaçóes entre alunos, docentes e profissionais, podendo contribuir para a qualidade da assistência e reflexão sobre o processo de trabalho.

Embora sejam poucas as experiências registradas de EAD em enfermagem, pode-se constatar que essa nova dinâmica de ensino é relevante, pois permite que o aluno e profissionais sejam capacitados e inseridos numa nova realidade virtual que permite a interatividade e o conhecimento de novas tecnologias. Além disto, é uma alternativa importante para superar as dificuldades encontradas na formação, capacitação e educação permanente dos profissionais de enfermagem, definidas pelo cenário da profissão no país $[3,12]$.

Em relação ao aperfeiçoamento tecnológico, no segundo semestre de 2011 e primeiro semestre de 2012 , respectivamente $53,2 \%$ e $62,5 \%$ dos alunos afirmam a necessidade de melhorar a conectividade, pois em muitos momentos acontece a interrupçáo ou queda da transmissão durante as videoconferências. Muitas vezes, os municípios não dispóem de uma banda de conexão adequada para uma efetiva transmissão de áudio e vídeo. Compreende-se que essa situação pode se traduzir em um fator que dificulta a capacitação dos alunos e profissionais, pois quando a infraestrutura tecnológica é inadequada, a atividade de videoconferência fica comprometida.

Outros aspectos apontados referem-se às dificuldades técnicas para o acesso ao sistema, ou seja, o espaço físico limitado para os equipamentos na Unidade Básica de Saúde (UBS), que geralmente estão localizados no consultório médico. Assim, ocorrem, concomitantemente, consultas médicas, $o$ atendimento de urgência e as videoconferências. Essas dificuldades caracterizam-se como pessoais, práticas e de organização. As barreiras pessoais são causadas por experiências negativas de aprendizado, assim como pela falta de motivação para aprender. As barreiras práticas, por sua vez, pela falta de tempo, 
pela falta de infraestrutura e pela falta de condição para acessar a tecnologia. Quanto às barreiras organizacionais, essas surgem quando a UBS não fornece os suportes necessários para a utilização das ferramentas do Projeto Telenfermagem [13].

Outro aspecto que merece ser discutido relaciona-se à contribuição e importância das videoconferências para os alunos e academia. No segundo semestre de 2011 e primeiro semestre de 2012 , respectivamente $86,7 \%$ e $100 \%$ dos alunos afirmam a importância dos temas discutidos nas videoconferências, o que reforça a aproximaçáo do conteúdo teórico com a prática, ou seja, da Universidade com o SUS e propicia a troca de saberes, numa proposta que integra a formação profissional e a sua práxis. As videoconferências são fonte de conhecimento e momento de resolução de dúvidas a respeito do tema abordado [14].

A possibilidade de discutir os temas das videoconferências, conforme a necessidade da equipe de saúde, estimula a capacidade autoformativa e de trabalho em uma equipe, podendo concorrer para o desenvolvimento pessoal, social profissional desse grupo. Dessa forma, o emprego da tecnologia constitui mais uma opção para o percurso formativo do aluno no espaço de trabalho, em prol da construçáo do seu conhecimento e aprendizagem [15]. Entende-se a importância de concatenar conhecimentos norteadores de uma proposta de ensino vinculada ao mundo do trabalho, tornando-se um desafio para concretizar o princípio da integralidade na prática social, política, ética e técnica [3].

Em relação às teleconsultorias, houve um incremento na utilização dessa ferramenta a partir do segundo semestre de 2011. Esta situação se justifica, considerando que a disciplina adotou efetivamente a teleconsultoria como instrumento regular no processo de ensino aprendizagem a fim de esclarecer os problemas clínicos, gerenciais, açóes de saúde ou questóes relativas ao processo de trabalho que se apresentavam no cotidiano do desenvolvimento da disciplina.

Os resultados apresentam fatores positivos que consolidam a efetividade do Projeto de Telenfermagem na disciplina Estágio Curricular I da EEUFMG, garantindo a parceria da Universidade com os serviços de saúde da capital e municípios de Minas Gerais, bem como a inserção do aluno no processo ensino aprendizagem, ao propiciar a identificação das açóes transformadoras ou vulneráveis nos diferentes cenários da APS, com uma posição crítica e reflexiva, contribuindo, assim, com a formação do enfermeiro.

\section{Conclusão}

O Projeto de Telenfermagem é de grande importância e eficácia, uma vez que é uma alternativa que colabora para superar as dificuldades encontradas na formação acadêmica, além de servir de suporte para a assistência à saúde oferecida pelos alunos da disciplina Estágio Curricular I/ Internato Rural e Metropolitano à comunidade, por meio da utilizaçáo da ferramenta de teleconsultoria e videoconferência.

O emprego das ferramentas do Projeto Telenfermagem possibilitou uma troca de experiência a partir da discussáo teórica e prática entre a Universidade e os serviços de saúde, com enfoque na APS, tanto para os alunos como para os profissionais. Neste sentido, as tecnologias da educação constituem um crescente recurso que permite um suporte pedagógico no processo do ensino aprendizagem na formação do enfermeiro.

O Projeto de Telenfermagem amplia o conhecimento na área da saúde, tornando disponível o acesso da tecnologia para a equipe de saúde, onde e quando for necessário, ajudando nas resoluçóes dos problemas de saúde para diferentes áreas de abrangência e realidades. Este recurso global contribui para o desenvolvimento e o aperfeiçoamento dos alunos e profissionais, contribuindo para que o processo de atenção à saúde resulte em benefícios, tanto para o usuário, como para aqueles que prestam assistência.

\section{Referências}

1. Nogueira DXP. A trajetória histórica das políticas educacionais brasileiras e o desenvolvimento da educação a distância no Brasil. [citado 2012 Ago 27]. Disponível em: URL: http://www.anpae.org.br/congressos_antigos/simposio2009/72.pdf.

2. Vilaça MLC. Educação a distância e tecnologias: conceitos, termos e um pouco de história. Revista Magistro 2010;1(2):89-101.

3. Camacho ACLF. Análise das publicaçôes nacionais sobre educação à distância na enfermagem. Rev Bras Enferm 2009;62(4):588-93.

4. Sarreta FO. Educação permanente em saúde para os trabalhadores do SUS [online]. Sáo Paulo: Editora UNESP; São Paulo: Cultura Acadêmica, 2009. 248 p.

5. Candido MCFS, Furegato ARF. Transtornos depressivos: um material didático para educação à distância. Esc Anna Nery Rev Enferm 2008;12(3):473-8. 
6. Brasil. Ministério da Saúde. Portaria 687 de 30 de março de 2006. Aprova a Política de Promoção da Saúde. [citado 2012 Set 1]. Disponível em URL: http:portal. saude.gov.br/portal/.../Política nacional_\%20saude_nv.pdf

7. Cruz ELD, Sanches LMP, Alves DS, Machiavelli J, Novaes M. A tele-enfermagem como ferramenta para capacitação do enfermeiro que atua no programa saúde da família. [citado 2012 Ago 24]. Disponível em URL: http://cbtms.com.br/congresso/trabalhos/021.pdf.

8. Seixas CA, Mendes IAC, Godoy S, Costa AL. Implantaçáo de sistema de videoconferência aplicado a ambientes de pesquisa e de ensino de enfermagem. Rev Bras Enferm 2004;57(5):620-4.

9. Santos WN. Telessaúde: um instrumento de suporte assistencial e educação permanente. Belo Horizonte: UFMG; 2006.

10. Santos SGF, Marques IR. Uso dos recursos de Internet na Enfermagem: uma revisão. Rev Bras Enferm 2006;59(2):212-6.

11. Wen CL. Telemedicina e Telessaúde - Um panorama no Brasil. Informática Pública 2008;10(2):7-15.
12. Guimaraes EMP, Godoy SCB, Ferreira CS, Melo CR, Azevedo NI. Desafios e Tendências na Implantação da Telenfermagem no Programa Nacional de Telessaude em Minas Gerais. [citado 2012 Set 15]. Disponível em URL: http://www.cbtms.com.br/congresso/trabalhos/081.pdf

13. Cortez RC, Ferreira AF, Coto JC. Fatores relevantes e dificultadores para o ensino e a aprendizagem a distância a partir da perspectiva dos alunos. [citado 2012 Nov 8]. Disponível em: URL: http://repositorio.ufsc.br

14. Ferreira MLSM, Cotta RMM, Lugarinho R, Oliveira MS. Construçáo de Espaço Social Unificado para Formação de Profissionais da Saúde no Contexto do Sistema Único de Saúde. Rev Bras Educ Med 2010;34(2):304-9.

15. Reis A. Tecnologias da Informação e da Comunicação na Educação em Saúde. O Caso da Formação em Enfermagem. Revista Latinoamericana de Tecnoligía Educativa - RELATEC 2009;8(1):105-25. 